\title{
Application Effect of Nursing Risk Management in Nursing of Kidney Diseases
}

\author{
Dongyun Zhi, Siyu Chen \\ Second people's Hospital of Yunnan, Kunming Yunnan, China, 650032
}

Keywords: Nursing risk management, kidney medical diseases, application effect

\begin{abstract}
Objective: To analyze the application effect of nursing risk management in the nursing of kidney diseases. Methods: Sixty patients with renal diseases treated in our hospital were selected as study subjects, and divided into two groups: control group and observation group, thirty people in each group. The control group used routine nursing methods, the observation group selected nursing risks management methods, and analysis of the condition of the two groups of patients after care. Results: After analysis and evaluation, the indicators of the observation group were higher than the control group; for the nursing satisfaction rate, the observation group was $96.6 \%$, and the control group was $91.6 \%$. Conclusion: Applying nursing risk management to the nursing of kidney medical diseases can further improve the quality of nursing services and patient satisfaction, which is worthy of clinical application.
\end{abstract}

\section{Introduction}

Unstable patient condition is very common in the nursing service process. The risk factors of nursing in the environment will aggravate the risk of nursing work and directly threaten the safety of hospitals, nursing staff and patients. In order to minimize the risk of care, it is necessary to use nursing risk management to solve, reduce risk factors and improve the quality of nursing services. The article selected 60 patients with renal diseases treated by the hospital as the research object and analyzed the effect of applying nursing risk management.

\section{The Significance of Implementing Nursing Risk Management}

Implement nursing risk management, strengthen nursing staff's risk awareness, improve risk judgment ability, reduce risk factors caused in the service process, and reduce the incidence of nursing risk events as much as possible. Protect the patient's life safety, avoid the doctor-patient disputes that have gone wrong during the nursing process, and improve the quality of care.

\subsection{Principles of Nursing Management}

With the help of nursing risk management, the mindset of caregivers can be transformed. From fear of error to hard work, managers gradually shift from handling error behaviors to controlling risk behaviors, raising risk management to safety management, paying attention to quality management, and controlling various potentials. Risk, prevent problems before they occur, and create a safe atmosphere. In this regard, new risk management is more comprehensive and reasonable than other management.

\subsection{Build a Preventive Care Risk System}

The professional management of nursing risk management is characterized by strong professional skills, large individual differences, and relatively complex diseases. In clinical activities, its management system is not sound enough. In this regard, increase medical risk management, find the deficiencies and problems in the system, formulate and implement normative standards to ensure medical safety and medical quality, but not to investigate the mistakes and responsibilities of the parties and individuals. The main systems for preventive medical risks in economically developed areas include clinical supervision systems, monitoring and guidance of clinical systems and work, continuing professional education and training systems, and accident reporting systems. 


\subsection{Patient-centered Service Objectives}

The main purpose of implementing nursing risk management is to avoid the occurrence of nursing errors, minimize patient losses, and improve the quality, safety and effectiveness of nursing services. The hospital should set the service as the core of the patient, provide quality services for patients, and put the patient's life and property safety in the first place, fully demonstrating the patient's status and life value.

\subsection{Promote the Continuous Improvement of Nursing Quality}

In the process of improving the quality of care, care risk management is one of the links, and the risk management plan must be consistent with the care quality assurance plan. After actively optimizing service attitudes, we will increase risk prevention education, pay attention to the management of unsafe factors in nursing details, reduce the occurrence of nursing mistakes as much as possible, reduce the loss of hospitals to this, avoid disputes between doctors and patients, and improve care. quality.

\section{Cognition of Nursing Risk Management}

\subsection{Understand the relationship between nursing risk and benefit}

Some hospital management scholars have to show that no matter what kind of clinical activities, even very simple or extremely small clinical activities, there will be certain risks. For example, although the caregiver turned over the patient on time according to the routine, there are still family members who report that the condition is aggravated because of turning over. When patients use intravenous chemotherapy drugs, it is not because of the low professional skills of the nursing staff, but the patients and their families still blame the caregivers. In this regard, when implementing a certain kind of nursing behavior, the nursing staff must fully consider the patient's experience to obtain the benefits and risks and evaluate them. Once the expected benefit-risk is too large, patients are advised to take these risks and treat them.

\subsection{Understand the Risks Caused by Human Factors}

Most people think that mistakes are due to the deviation of consciousness and thinking ability. Once the feelings of fatigue, tiredness, nervousness, etc. are not concentrated, the operation process will be problematic. In the hospital system, medical device operation and medical service practices are dynamic processes, and there are some risks to their personnel, equipment and services. For example, the electric knife and electrode plate used in the operation process burn the patient; the sudden failure of the call system delays the patient's rescue time; the ground wetness causes the patient to fall. Regarding the risk of human factors and system factors, it is necessary to choose a reasonable solution to avoid risks in the nursing process and ensure safety.

\subsection{Patient Behavior is One of the Risk Factors of Care}

Nursing work is a management activity in which both patients and nurses participate together. The normal development of nursing activities depends on the close cooperation and support of patients. The patient's motivation and behavior of seeking medical care have an important impact on whether the patient cooperates closely with the medical staff and actively participates in the treatment of the disease. If the patient understands his or her condition and cooperates fully with the medical staff, the actual benefits in the medical process will increase significantly. If the patient has risky behavior, an unhealthy lifestyle, or adopts a non-cooperative attitude, such as suicide and self-harm, the risk of the nursing process will rise.

\section{Information and Methods of Nursing Risk Management in the Nursing of Kidney Diseases}

Sixty patients with renal diseases treated in our hospital were selected as the study subjects, and divided into two groups: control group and observation group, 30 in each group, 13 male patients in 
the control group and 17 female patients; There were 20 male patients in the observation group and 10 female patients. After communication, they voluntarily joined the application analysis. The control group selected the routine nursing method, and the observation team selected the nursing risk management method. The main contents were as follows:

\subsection{Nursing Risk Assessment}

Iatrogenic factors and patient factors are the most common risk factors for kidney medical care. Among the medical source factors, the workload of the nursing staff is generally causing problems in the work; the safety protection work of the ward is unreasonable, causing the patient to fall into bed and fall many times; the comprehensive quality of the nursing staff and the supervision and supervision work are not in place, and the business level is poor. There is a lack of rationality in the management of nursing. Due to the poor supervision work, problems arise in the work, especially the inspection and management of materials, instruments and medicines, which ultimately leads to the problem of nursing operation. There is not enough understanding of legal knowledge education, and there is a lack of understanding of legal knowledge in the work. For example, if the patient's right to know and notice are not timely, especially the drainage tube and tracheal intubation, the treatment funds are not immediately notified to the patient. The patient's factors, the diuretics used by the patient due to their own diseases, accompanied by the decline of the elderly's body function, high blood pressure and activity disorders are easy to fall; The body's nutrient loss and edema, making patients easy to form pressure sores; Hemodialysis patients are prone to adverse events caused by problems such as pipe shedding, clogging and infection; Because of limited economic conditions, high treatment costs lead to increased psychological stress; Diseases can easily cause a variety of complications, and the symptoms are undulating, making Security risks occur frequently.

\subsection{Nursing Risk Management Program}

Under the premise of knowing the risk factors, the protection management should be done according to reasonable risk factors. In the management process, to ensure the standardization and process of management work, all work has a system that can be referenced to provide guarantee for the orderly implementation of work. The work assessment system, recruitment system, supervision system, incentive system and training system are used to optimize the standard of nursing management, so that the comprehensive quality of nursing staff can meet the work requirements and stimulate the enthusiasm of the nursing staff. In the training management, we should not only pay attention to the nursing staff's own technology, but also interact with the patients, guide them to understand the legal common sense and psychological content, and strengthen the ability of the nursing staff to respond to emergencies. Reasonable use of supervision mechanisms to strengthen the dynamic supervision and inspection of nursing work, such as checking the information reported by nursing work, the dynamics of nursing operations and the examination of nursing theory knowledge. Comprehensively consider the supervision mechanism, strengthen the investigation of patient complaints and feedback, and then make targeted adjustments. Grasp all aspects of evaluation and work operation evaluation, and provide data basis for work evaluation indicators. Regularly educate and study patients, familiarize each patient with various issues during hospitalization, improve coordination, and provide psychological counseling and construction for patients to help their patients recover. Select resource optimization configuration to complete staffing work, allocate tasks reasonably, prevent overloading and maintain flexible work control. Regularly distribute salary and benefits to employees to stimulate the enthusiasm of nursing staff.

\subsection{Evaluation Observation}

The nursing service quality assessment and patient care satisfaction status of the two groups of patients were observed, and they were satisfied, generally satisfied, and dissatisfied to form a satisfaction rating system. Satisfaction rate $=$ very satisfactory rate + general satisfaction rate. Basic care services, quality of disinfection management, and ward care management are important components of care service quality. The higher the score, the better the quality. 


\section{Comparison of Nursing Results between the Control Group and the Observation Group}

\subsection{Quality of Care Service scores}

As shown in Table 1, in the nursing service quality score, the observation group was significantly higher than the control group.

Table 1 Two groups of nursing service quality score status map

\begin{tabular}{|l|c|c|c|c|}
\hline Group & Number & $\begin{array}{c}\text { Basic Care } \\
\text { Service }\end{array}$ & $\begin{array}{c}\text { Quality of Disinfection } \\
\text { Management }\end{array}$ & $\begin{array}{c}\text { Ward Care } \\
\text { Management }\end{array}$ \\
\hline Control group & 30 & $94.6 \pm 2.4$ & $96.9 \pm 1.5$ & $99.2 \pm 0.5$ \\
\hline Observation group & 30 & $85.6 \pm 1.7$ & $90.0 \pm 1.1$ & $90.1 \pm 2.1$ \\
\hline
\end{tabular}

\subsection{Care Satisfaction of the Two Groups of Patients}

Table 2 two groups of patient care satisfaction service

\begin{tabular}{|l|c|c|c|c|c|}
\hline Group & Number & Very Satisfied & $\begin{array}{c}\text { General } \\
\text { Satisfaction }\end{array}$ & Dissatisfaction & Total Satisfaction Rate \\
\hline Control group & 30 & $55.6 \%$ & $36 \%$ & $1 \%$ & $91.6 \%$ \\
\hline Observation group & 30 & $60.8 \%$ & $35.8 \%$ & $3 \%$ & $96.6 \%$ \\
\hline
\end{tabular}

As shown in Table 2, for the satisfaction of nursing, the observation group was $96.6 \%$, and the control group was $91.6 \%$.

\section{Analysis of the Application Effect of Nursing Risk Management in the Nursing of Kidney Diseases}

In recent years, risk management nursing has been widely used in the nursing of kidney diseases. In the process of nursing work, the patient's risk is effectively evaluated. According to the evaluation results, a rationalization plan is formulated to further improve the quality of care, reduce the risk of patients, and clear the risk. Effectively prevent and predict the emergence of risks. In the clinical application of nursing risk management, including nursing risk assessment, nursing risk identification and nursing risk assessment, nursing staff must meet clinical standards, strengthen nursing risk management techniques, and implement integrated management in the system and training awareness. In the nursing work, the risk occurrence probability of the patient can be reduced, the patient's satisfaction with the nursing service can be improved, the occurrence of the malignant event can be reduced, the patient can be more communicated and communicated, and the relationship between the doctor and the patient can be coordinated. According to the above discussion, after the implementation of nursing risk management, the medical staff of the Department of Nephrology has a deeper understanding of risk management, and the risk factors are ideological and improve the orientation of risk actions. By comparison, the risk of non-care risk management is higher than that of nursing care management, and the satisfaction of the two groups is relatively obvious. All of the above have verified the importance of risk management in the nursing of kidney diseases. The more satisfied you are with the care, the lower the risk of taking it, which is important for both the caregiver and the patient.

\section{Conclusion}

In summary, risk management is a management process that evaluates, judges, and resolves existing medical risks and minimizes the occurrence of medical risk events. Since the introduction of nursing risk management, the quality of each nursing has increased, the degree of patient satisfaction with hospital care services has also been significantly improved, and medical disputes have been reduced, which is worthy of clinical application. 


\section{References}

[1] Ke Wu. Analysis of the Application Effect of Nursing Risk Management in the Nursing of Kidney Diseases[J]. Chinese and Foreign Women's Health Research, 2016, (16): 13-15.

[2] Lan Deng, Rujuan Bao, Qinghua Liu. Risk Factors Analysis and Risk Management Evaluation of Kidney Medical Diseases[J]. Pharmaceutical Frontier, 2016, (27): 228-229.

[3] Lee Liu, Na Sun, Pingping Sun. Analysis of the Effect of Risk Management in the Nursing of Renal Medicine[J]. Chinese Journal of Health Nutrition, 2015, (5): 116-117.

[4] Huiqin Yu, Shaojuan Liu. Analysis of the Effect of Nursing Risk Management on the Safety Management of Patients with Nephrology[J]. Clinical and Educational Sciences, 2016, (2): 234-236.

[5] Hua Ma. Nursing Analysis of Kidney Medical Diseases[J]. Health Care Guide, 2017, (19): 88-89.

[6] Biyan Jiang, Hong Zhu, Xiaoli Sun. Practice and Experience of Internal Nursing Risk Management [J]. Journal of Traditional Chinese Medicine Management, 2017, (11): 108-110. 